\title{
ON BLD-MAPPINGS WITH SMALL DISTORTION
}

\author{
AAPO KAURANEN, RAMI LUISTO, AND VILLE TENGVALL \\ Dedicated to Professor Pekka Koskela on his 59th birthday
}

\begin{abstract}
We show that every $L$-BLD-mapping in a domain of $\mathbb{R}^{n}$ is a local homeomorphism if $L<\sqrt{2}$ or $K_{I}(f)<2$. These bounds are sharp as shown by a winding map.
\end{abstract}

\section{INTRODUCTION}

Mappings with $L$-bounded length distortion (abbr. $L$-BLD mappings) were originally introduced by Martio and Väisälä in MV88 as continuous, sense-preserving, discrete, and open mappings

$$
f: \Omega \rightarrow \mathbb{R}^{n} \quad\left(\Omega \subset \mathbb{R}^{n} \text { domain with } n \geq 2\right)
$$

satisfying the upper and lower length distortion bounds

$$
L^{-1} \ell(\alpha) \leq \ell(f \circ \alpha) \leq L \ell(\alpha)
$$

for every path $\alpha$ in $\Omega$ and for a fixed constant $L \geq 1$, where $\ell(\gamma)$ denotes the length of a path $\gamma$. Notice that no constant map satisfies (11). These mappings form a superclass of local $L$-bi-Lipschitz mappings which have been studied by several authors, see e.g. [DP15, HMZ18, LP14, HS02] and the references therein. If a mapping is an $L$-BLD-mapping for some $L \geq 1$ we may simply call it a $B L D$-mapping.

Unlike local bi-Lipschitz maps, BLD-mappings do not need to be local homeomorphisms. Indeed, for instance the map

$$
(r, \theta, z) \stackrel{w}{\mapsto}\left(\sqrt{2}^{-1} r, 2 \theta, \sqrt{2}^{-1} z\right) \quad\left(z \in \mathbb{R}^{n-2}\right)
$$

in cylindrical coordinates of $\mathbb{R}^{n}$ defines a noninjective $\sqrt{2}$-BLD mapping. Our first theorem shows that this mapping is extremal for the failure of local homeomorphism property:

Theorem 1. Every L-BLD-mapping

$$
f: \Omega \rightarrow \mathbb{R}^{n} \quad\left(\Omega \subset \mathbb{R}^{n} \text { domain with } n \geq 2\right)
$$

with $L<\sqrt{2}$ is a local homeomorphism.

Date: February 13, 2020.

2010 Mathematics Subject Classification. 57M12, 30C65.

Key words and phrases. BLD-mappings, branch set, quasiregular mappings, local homeomorphism.

A.K. acknowledges the support of Academy of Finland, grant number 322441.

R.L. was partially supported by the Academy of Finland (grant 288501 'Geometry of subRiemannian groups') and by the European Research Council (ERC Starting Grant 713998 GeoMeG 'Geometry of Metric Groups').

The research of V.T. was supported by the Academy of Finland, project number 308759. 
Mappings of $L$-bounded length distortion form also a subclass of $L^{2(n-1)}$ quasiregular mappings, see MV88, Lemma 2.3]. We recall that a mapping

$$
f: \Omega \rightarrow \mathbb{R}^{n} \quad\left(\Omega \subset \mathbb{R}^{n} \text { domain with } n \geq 2\right)
$$

is called $K$-quasiregular if it belongs to Sobolev space $W_{l o c}^{1, n}\left(\Omega, \mathbb{R}^{n}\right)$ and satisfies the following distortion inequality

$$
|D f(x)|^{n} \leq K J_{f}(x) \quad \text { a.e. }
$$

for a given constant $K \geq 1$. Here and what follows $|A|$ stands for the operator norm of a given $n \times n$ matrix $A$ and

$$
J_{f}(x)=\operatorname{det} D f(x)
$$

denotes the Jacobian determinant of the differential matrix $D f(x)$. To every quasiregular mapping we associate the well-known inner and outer distortion functions defined as follows

$$
K_{I}(x, f)= \begin{cases}\frac{\left|D^{\#} f(x)\right|^{n}}{J_{f}(x)^{n-1}}, & \text { if } J_{f}(x)>0 \\ 1, & \text { otherwise }\end{cases}
$$

and

$$
K_{O}(x, f)= \begin{cases}\frac{|D f(x)|^{n}}{J_{f}(x)}, & \text { if } J_{f}(x)>0 \\ 1, & \text { otherwise, }\end{cases}
$$

measuring how the infinitesimal geometry of $n$-dimensional balls is distorted by the mapping. Here and what follows

$$
D^{\sharp} f(x)=(\operatorname{cof} D f(x))^{T},
$$

where cof stands for cofactor matrix. The corresponding inner and outer dilatations are defined by

$$
K_{I}(f):=\operatorname{esssup}_{x \in \Omega} K_{I}(x, f), \quad \text { and } \quad K_{O}(f):=\operatorname{ess~sup}_{x \in \Omega} K_{O}(x, f) .
$$

For the theory and basic properties of quasiregular mappings we refer to the standard monographies [M01, Ric93, Res89, Vuo88.

By the generalized Liouville theorem of Gehring [Geh62] and Reshetnyak [Res67] non-constant 1-quasiregular mappings are restrictions of Möbius transformations. On the other hand, for the winding map in (2) we have

$$
K_{I}(w)=2 \quad \text { and } \quad K_{O}(w)=2^{n-1} .
$$

In [MRV71, Remark 4.7] it was conjectured that any quasiregular mapping with $K_{I}(f)<2$ is a local homeomorphism. That is, the behaviour of the winding map is extremal in this sense.

The conjecture is not yet solved, but some partial results are known. By the result of Martio, Rickman, and Väisälä [MRV71, Theorem 4.6] there exists $\varepsilon(n)>0$ such that every quasiregular mapping

$$
f: \Omega \rightarrow \mathbb{R}^{n} \quad\left(\Omega \subset \mathbb{R}^{n} \text { with } n \geq 3\right) \quad \text { with } K_{I}(f)<1+\varepsilon(n)
$$

is a local homeomorphism, see also Gol71]. The best known quantitative bound for the number $\varepsilon(n)>0$ is given by Rajala in Raj05. Furthermore, the conjecture is known to be true when the branch set

$$
B_{f}=\{x \in \Omega: f \text { is not a local homeomorphism at } x\}
$$


of the mapping is geometrically nice. In particular, this is the case when the branch set contains a rectifiable curve, see e.g. [Ric93, p. 76]. We give a short proof showing that the conjecture holds also for $L$-BLD mappings. See also [HK00] for other related injectivity results for BLD mappings.

Theorem 2. Let

$$
f: \Omega \rightarrow \mathbb{R}^{n} \quad\left(\Omega \subset \mathbb{R}^{n} \text { domain with } n \geq 2\right)
$$

be an L-BLD-mapping. Then

$$
K_{I}(f) \geq i(x, f) \quad \text { for every } x \in \Omega .
$$

Especially, if $K_{I}(f)<2$ then $f$ is a local homeomorphism.

Notice that the conclusion of Theorem 1 does not hold for quasiregular mappings in general. In [MRV71 there is an example of a quasiregular mapping $f: \Omega \rightarrow \mathbb{R}^{n}$ for which $\sup \{i(x, f): x \in \Omega\}=\infty$. Also, notice that Theorem 1 is valid in dimension two but the same conclusion does not hold for planar quasiregular mappings as one can see by considering the holomorphic function

$$
f: \mathbb{C} \rightarrow \mathbb{C}, \quad f(z)=z^{2} .
$$

This function is obviously not a BLD-mapping. We also point out that the proof of Theorem 2 is based on the modulus of continuity of quasiregular mappings and on the $L$-radiality of BLD-mappings. The BLD-property is used here only for the $L$-radiality.

Finally, we recall that by Zorich's theorem [Zor67 every locally injective, entire quasiregular mapping in dimension $n \geq 3$ is quasiconformal. Zorich's theorem fails in the plane, which is shown by the mapping $z \mapsto \exp (z)$. Note that in the BLD-setting every entire, locally homeomorphic BLD-mapping in $\mathbb{R}^{n}, n \geq 2$, is a bi-Lipschitz homeomorphism onto $\mathbb{R}^{n}$. See eg. MV88, Lemma 4.3]. If we combine this with our main results we obtain the following:

Corollary 4. Let $f: \mathbb{R}^{n} \rightarrow \mathbb{R}^{n}, n \geq 2$, be an entire BLD-mapping with

$$
L<\sqrt{2} \text { or } K_{I}(f)<2 .
$$

Then $f$ is an L-bi-Lipschitz map onto $\mathbb{R}^{n}$.

\section{Proof of Theorem 1}

In the proof of Theorem 1 we use the $L$-radiality property of mappings with $L$-bounded length distortion, see [Lui17]. For a space $X$ we use the following notation:

$$
\begin{aligned}
& H_{j}(X)=\text { " } j \text { th homology group of } X " \\
& H^{j}(X)=\text { " } j \text { th Alexander-Spanier co-homology group of } X " .
\end{aligned}
$$

For definition and basic properties of these homology and co-homology groups, as well as the suspension mappings used in the following proof, we refer to [Hat02]. Necessary information on the topological degree theory used in the proof can be found from [Ric93, Chapter I]. 
Proof of Theorem 1. Let $f: \Omega \rightarrow \mathbb{R}^{n}$ be an $L$-BLD-mapping with $L<\sqrt{2}$. For the contradiction, suppose that the branch set

$$
B_{f}=\{x \in \Omega: f \text { is not a local homeomorphism at } x\}
$$

of $f$ is nonempty and fix a point $x_{0} \in B_{f}$. Without loss of generality we may assume that $x_{0}=\mathbf{0}=f\left(x_{0}\right)$.

We take first the blow-up of $f$ by defining a sequence of mappings

$$
g_{j}: j \Omega \rightarrow \mathbb{R}^{n}, \quad g_{j}(x)=j f(x / j),
$$

where

$$
j \Omega:=\left\{j x \in \mathbb{R}^{n} \mid x \in \Omega\right\} \quad(j=1,2, \ldots) .
$$

We note that by [MV88, Theorem 4.7] (see also [Lui17, Section 4]) the mappings $g_{j}$ are $L$-BLD-mappings and contain a subsequence converging uniformly to an $L$-BLD-mapping $g: \mathbb{R}^{n} \rightarrow \mathbb{R}^{n}$ such that

$$
\mathbf{0} \in B_{g}, \quad g(\mathbf{0})=\mathbf{0}, \quad \text { and } \quad g^{-1}(\{g(\mathbf{0})\})=\mathbf{0} .
$$

Furthermore, since the $L$-BLD-mapping $f$ is $L$-radial at $\mathbf{0}$, that is, there exists $r_{0}>0$ such that for every $x \in B\left(0, r_{0}\right)$ we have

$$
L^{-1}\|x\| \leq\|f(x)\| \leq L\|x\| .
$$

Clearly, for $g_{j}$ we have

$$
L^{-1}\|x\| \leq\left\|g_{j}(x)\right\| \leq L\|x\|
$$

for all $x \in B\left(0, j r_{0}\right)$.

This implies that the blow-up mapping $g$ satisfies

$$
L^{-1}\|x\| \leq\|g(x)\| \leq L\|x\|
$$

for all $x \in \mathbb{R}^{n}$. In particular, we note that the composition of $\left.g\right|_{\partial B(\mathbf{0}, r)}$ and the radial projection map

$$
p: \mathbb{R}^{n} \backslash B(\mathbf{0}, r / L) \rightarrow \partial B(\mathbf{0}, r / L), \quad p(x)=(r / L) \frac{x}{\|x\|}
$$

is $L$-Lipschitz. Thus, the mapping

$$
h: \mathbb{S}^{n-1} \rightarrow \mathbb{S}^{n-1}, \quad h(x)=(L / r)\left(\left.p \circ g\right|_{\partial B(\mathbf{0}, r)}\right)(r x)
$$

is $L^{2}$-Lipschitz. Now by a classical dilatation result Gro99, Proposition 2.9, p. 30] under the assumption $L<\sqrt{2}$ we see that the induced homomorphism

$$
h_{*}: H_{n-1}\left(\mathbb{S}^{n-1}\right) \rightarrow H_{n-1}\left(\mathbb{S}^{n-1}\right)
$$

equals either \pm id or the constant map.

Since for all $r>0$ the image of the restriction $\left.g\right|_{\partial B(\mathbf{0}, r)}$ avoids the origin, we note that the restrictions are in fact mutually homotopic in $\mathbb{R}^{n} \backslash\{0\}$ and homotopic to $h$. In particular since $\mathbb{S}^{n-1}$ is a homotopy retract of $\mathbb{R}^{n} \backslash\{0\}$, we see that $\left.g\right|_{\mathbb{R}^{n} \backslash\{0\}}$ is homotopic to $h \times \operatorname{id}_{\mathbb{R}}$ with the identification $\mathbb{R}^{n} \backslash\{0\} \simeq \mathbb{S}^{n-1} \times \mathbb{R}$.

Finally we note that as a BLD-mapping from $\mathbb{R}^{n}$ to $\mathbb{R}^{n}$, the mapping $g$ extends into a branched cover $\hat{g}: \mathbb{S}^{n} \rightarrow \mathbb{S}^{n}$, see e.g. [Lui16. Furthermore, by the above arguments this mapping $\hat{g}$ is homotopic to the suspension of the map $h$. Therefore, the induced homomorphism

$$
\hat{g}_{*}: H_{n}\left(\mathbb{S}^{n}\right) \rightarrow H_{n}\left(\mathbb{S}^{n}\right)
$$


equals either \pm id or the constant map. But now by the universal coefficient theorem [Hat02, p.190] the induced homomorphism

$$
\hat{g}^{*}: H^{n}\left(\mathbb{S}^{n}\right) \rightarrow H^{n}\left(\mathbb{S}^{n}\right)
$$

also equals either \pm id or a constant map. As the degree of a branched cover $\mathbb{S}^{n} \rightarrow \mathbb{S}^{n}$ is always non-zero, this implies that $\hat{g}$ has degree \pm 1 . Thus, the mapping $\hat{g}: \mathbb{S}^{n} \rightarrow \mathbb{S}^{n}$ is injective. This is a contradiction with the assumption $\mathbf{0} \in B_{f}$ and so the original claim holds.

\section{Proof of Theorem 2}

In what follows, for a given continuous mapping

$$
f: \Omega \rightarrow \mathbb{R}^{n} \quad\left(\Omega \subset \mathbb{R}^{n} \text { domain with } n \geq 2\right)
$$

we denote its local (topological) index at a point $x \in \Omega$ by $i(x, f) \in \mathbb{Z}$. We recall that as a sense-preserving, continuous, discrete and open mapping every BLD-mapping satisfies

$$
i(x, f) \geq 1 \quad \text { for every } x \in \Omega \text {. }
$$

Moreover, for a given point $x \in \Omega$ we have $i(x, f)=1$ if and only if $f$ is a local homeomorphism. Thus, in order to proof Theorem 2 it suffices to show that under the assumptions of the theorem we have $K_{I}(f) \geq i(x, f)$ for every $x \in \Omega$. For the properties of local index used here and what follows, see [Ric93, Chapter 1].

Proof of Theorem Q . Fix a point $x_{0} \in \Omega$ and denote

$$
\mu:=\left(i\left(x_{0}, f\right) / K_{I}(f)\right)^{\frac{1}{n-1}} .
$$

By combining [MV88, Corollary 2.13] and [Ric93, Theorem III.4.7, p.72] we see that there exists a radius $r>0$ and a constant $B \geq 1$ such that

$$
L^{-1}\left\|x_{0}-y\right\| \leq\left\|f\left(x_{0}\right)-f(y)\right\| \leq B\left\|x_{0}-y\right\|^{\mu}
$$

for every $y \in B\left(x_{0}, r\right)$. By letting $y \rightarrow x_{0}$ we get from (5) that $\mu \leq 1$. Especially, from here it follows that

$$
K_{I}(f) \geq i(x, f) \quad \text { for every } x \in \Omega,
$$

and the claim follows.

Remarks 7 . We end this note with the following remarks:

(a) In the planar case Theorem 2 implies Theorem 1, Indeed, if

$$
f: \Omega \rightarrow \mathbb{R}^{2} \quad\left(\Omega \subset \mathbb{R}^{2} \text { domain }\right)
$$

is an $L$-BLD-mapping with $L<\sqrt{2}$ then by [MV88, Lemma 2.3] it is $K$-quasiregular with

$$
K \leq L^{2(n-1)}<2 .
$$

Especially, in the planar case we have

$$
K_{I}(f)=K_{O}(f)<2 .
$$

Thus, by Theorem 2 the mapping $f$ is a local homeomorphism. 
(b) We do not know what is the optimal outer dilatation bound for the local injectivity of BLD-mappings. However, the map

$$
(r, \theta, z) \mapsto(r, 2 \theta, 2 z) \quad\left(z \in \mathbb{R}^{n-2}\right)
$$

in cylindrical coordinates would suggest that every BLD-mapping with $K_{O}(f)<2$ is a local homeomorphism.

(c) In [KLT] we obtained that for every $n \geq 2$ there exists a quasiregular mapping of the unit ball with a compact and nonempty branch set. Moreover, this mapping can be actually assumed to be a BLD-map. It would be interesting to find the optimal length distortion and outer and inner dilatation bounds for the existence of nonempty compact branch sets for BLD-mappings of the unit ball.

\section{REFERENCES}

[DP15] D. Drasin and P. Pankka. Sharpness of Rickman's Picard theorem in all dimensions. Acta Math., 214(2):209-306, 2015.

[Geh62] F. W. Gehring. Rings and quasiconformal mappings in space. Trans. Amer. Math. Soc., 103:353-393, 1962.

[Gol71] V. M. Gol'dšteln. The behavior of mappings with bounded distortion when the distortion coefficient is close to one. Sibirsk. Mat. Ž., 12:1250-1258, 1971.

[Gro99] M. Gromov. Metric structures for Riemannian and non-Riemannian spaces, volume 152 of Progress in Mathematics. Birkhäuser Boston Inc., Boston, MA, 1999.

[Hat02] A. Hatcher. Algebraic topology. Cambridge University Press, Cambridge, 2002.

[HK00] J. Heinonen and T. Kilpeläinen. BLD-mappings in $W^{2,2}$ are locally invertible. Math. Ann., 318(2):391-396, 2000.

[HMZ18] P. Hajłasz, S. Malekzadeh, and S. Zimmerman. Weak BLD mappings and Hausdorff measure. Nonlinear Anal., 177(part B):524-531, 2018.

[HS02] J. Heinonen and D. Sullivan. On the locally branched Euclidean metric gauge. Duke Math. J., 114(1):15-41, 2002.

[IM01] T. Iwaniec and G. Martin. Geometric function theory and non-linear analysis. Oxford Mathematical Monographs. The Clarendon Press, Oxford University Press, New York, 2001.

[KLT] A. Kauranen, R. Luisto, and V. Tengvall. Mappings of finite distortion: compactness of the branch set. (to appear in J. Anal. Math.), arXiv:1709.08724v3.

[LP14] E. Le Donne and P. Pankka. Closed BLD-elliptic manifolds have virtually Abelian fundamental groups. New York J. Math., 20:209-216, 2014.

[Lui16] R. Luisto. Note on local-to-global properties of BLD-mappings. Proc. Amer. Math. Soc., 144(2):599-607, 2016.

[Lui17] R. Luisto. A characterization of BLD-mappings between metric spaces. J. Geom. Anal., 27(3):2081-2097, 2017.

[MRV71] O. Martio, S. Rickman, and J. Väisälä. Topological and metric properties of quasiregular mappings. Ann. Acad. Sci. Fenn. Ser. A I, (488):31, 1971.

[MV88] O. Martio and J. Väisälä. Elliptic equations and maps of bounded length distortion. Math. Ann., 282(3):423-443, 1988.

[Raj05] K. Rajala. The local homeomorphism property of spatial quasiregular mappings with distortion close to one. Geom. Funct. Anal., 15(5):1100-1127, 2005.

[Res67] J. G. Reshetnyak. Liouville's conformal mapping theorem under minimal regularity hypotheses. Sibirsk. Mat. Ž., 8:835-840, 1967.

[Res89] Y. G. Reshetnyak. Space mappings with bounded distortion, volume 73 of Translations of Mathematical Monographs. American Mathematical Society, Providence, RI, 1989. Translated from the Russian by H. H. McFaden.

[Ric93] S. Rickman. Quasiregular mappings, volume 26. Springer-Verlag, Berlin, 1993.

[Vuo88] M. Vuorinen. Conformal geometry and quasiregular mappings, volume 1319 of Lecture Notes in Mathematics. Springer-Verlag, Berlin, 1988. 
[Zor67] V. A. Zorič. M. A. Lavrent'ev's theorem on quasiconformal space maps. Mat. Sb. (N.S.), 74 (116):417-433, 1967.

Department of Mathematics and Statistics, P.O. Box 35, FI-40014 UniverSITY OF JYVÄSKYLÄ, FinLAND

E-mail address: aapo.p.kauranen@jyu.fi

Department of Mathematics and Statistics, P.O. Box 35, FI-40014 UniverSITY OF JyvÄSKYLÄ, Finland

E-mail address: rami.luisto@gmail.com

Department of Mathematics and Statistics, P.O. Box 68 (Pietari Kalmin Katu 5), FI-00014 University of Helsinki, Finland

E-mail address: ville.tengvall@helsinki.fi 